\title{
Lung Pattern Classification for Interstitial Lung Diseases Using a Deep Convolutional Neural Network
}

\author{
Andreas Christe ${ }^{1}$, Marios Anthimopoulos ${ }^{2}$, Stergios Christodoulidis ${ }^{2}$, Lukas Ebner ${ }^{1}$, \\ Stavroula Mougiakakou ${ }^{2}$ \\ ${ }^{1}$ Department of Radiology, Inselspital, Bern University Hospital, University of Bern, \\ Switzerland \\ ${ }^{2}$ University of Bern, ARTORG Center for Biomedical Engineering Research Diabetes \\ Technology Research Group Murtenstrasse 50, CH-3008 Bern
}

Purpose: Deep learning techniques have recently achieved impressive results in a variety of computer vision problems, raising expectations that they might be applied in other domains, such as medical image analysis. We propose and evaluate a convolutional neural network (CNN), designed for the classification of interstitial lung disease (ILD) patterns.

Materials and methods: The proposed network consists of 5 convolutional layers with $2 \times 2$ kernels and LeakyReLU activations, followed by average pooling with size equal to the size of the final feature maps and three dense layers. The last dense layer has 7 outputs, equivalent to the classes considered: healthy, ground glass opacity (GGO), micronodules, consolidation, reticulation, honeycombing and a combination of GGO/reticulation (Fig. 1). To train and evaluate the CNN, we used a dataset of 14696 image patches, derived by 120 CT scans from different scanners and hospitals (Fig. 2). A comparative analysis proved the effectiveness of the proposed CNN against previous methods in a challenging dataset.

Results: The classification performance ( $\sim 85.5 \%)$ demonstrated the potential of CNNs in analyzing lung patterns. Pattern-sensitivities reached from $99 \%$ (consolidation) to $69 \%$ (honeycombing). The individual "true positive" and "false negative" results for each pattern is demonstrated in Fig. 3.

Conclusion: The CNN showed very promising results in lung pattern recognition outperforming many state-of-the-art methods. Future work includes, extending the CNN to three-dimensional data provided by CT volume scans.

Clinical Relevance: Integrating the proposed method into a CAD system helps providing a differential diagnosis for ILDs as a supportive tool for radiologists.
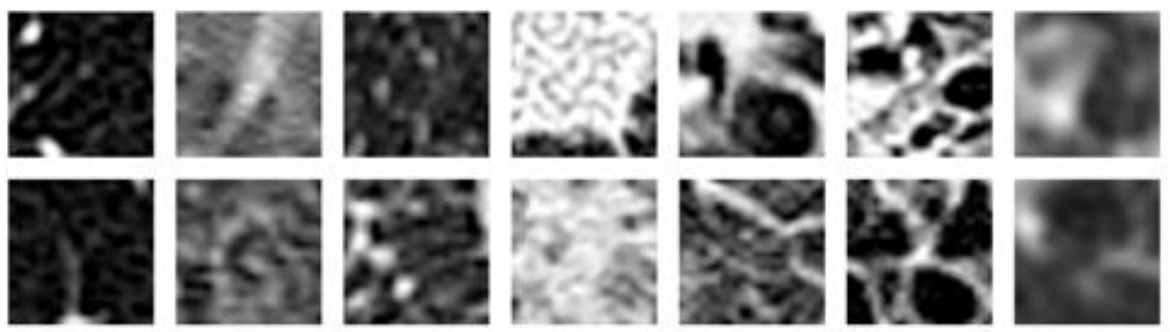

Fig 1. Examples of healthy tissue and typical ILD patterns from left to right: healthy, GGO, micronodules, consolidation, reticulation, honeycombing, combination of GGO and reticulation. 


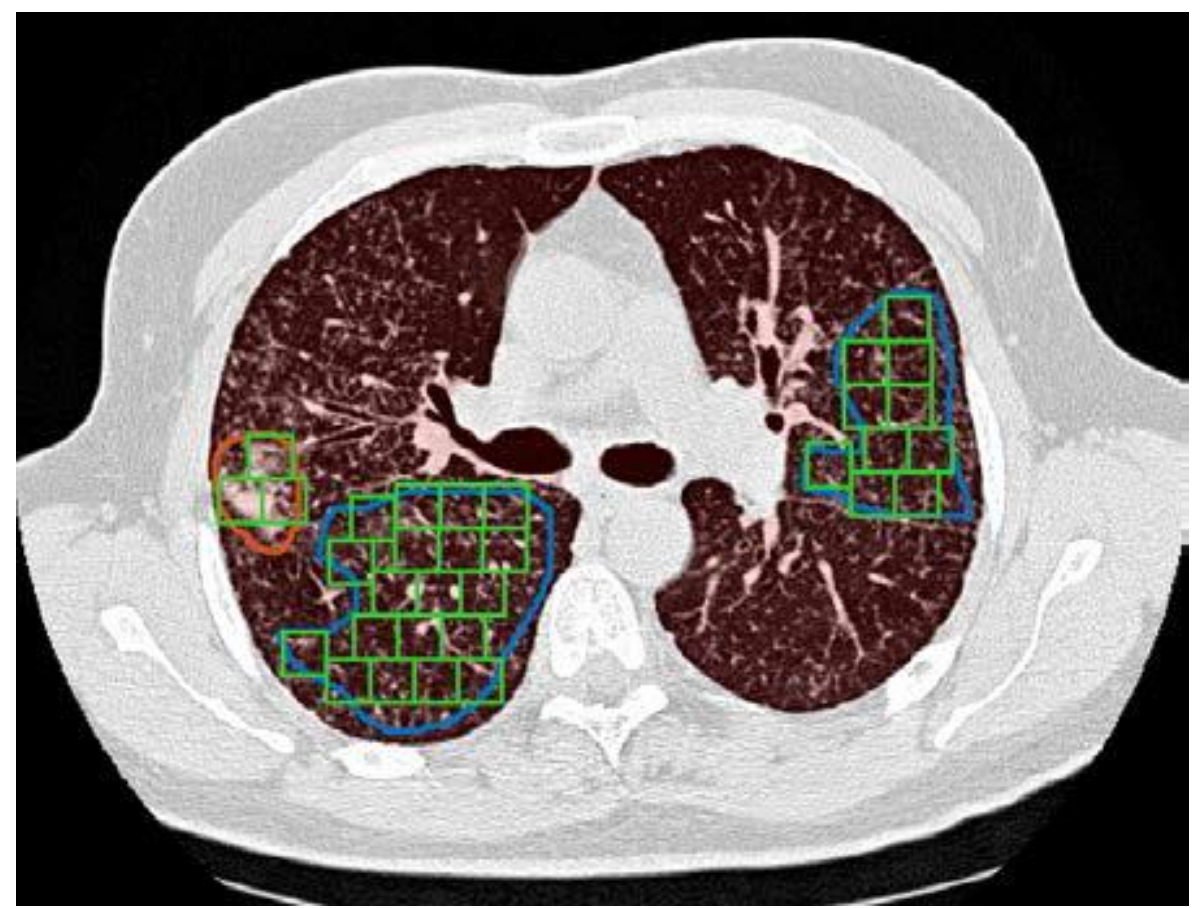

Fig 2. Example of generating image patches through the annotations of a CT slice. The lung field is displayed with transparent red. The polygons are the ground truth areas with considered pathologies. The patches have 100\% overlap with the lung, at least $80 \%$ overlap with the ground truth and $0 \%$ overlap with each other.

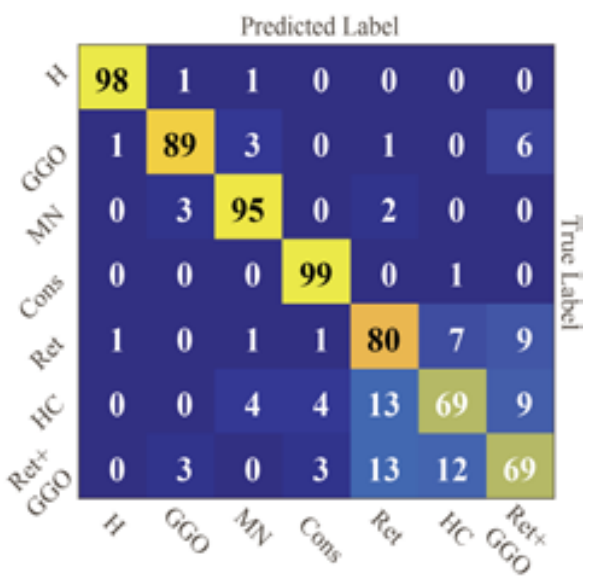

Confusion matrices of CNN. The entry in the $i^{\text {th }}$ row and $j^{\text {th }}$ column corresponds to the percentage of samples from class i that were classified as class j. H: healthy tissue; $\mathrm{MN}$ : micronodules; GGO: ground glass opacity; Cons: consolidation; Ret: reticulation, HC: honeycombing. 